\title{
Atrial Septal Defects: Special Considerations in Management and Closure in the Adult Patient Population
}

\author{
Jonathan Buber, MD, John P. Cheatham, MD, Darren P. Berman, MD* \\ The Heart Center, Nationwide Children's Hospital, The Ohio State University, Columbus, OH, USA
}

\begin{abstract}
Atrial septal defects are the third most common type of congenital heart disease, and include several types of atrial communications that allow shunting of blood between the systemic and the pulmonary circulations. Common manifestations in adult patients with untreated defects include exercise intolerance and atrial tachyarrhythmias. If remained untreated, right ventricular dysfunction, and pulmonary hypertension may ensue, leading to a lower quality of life and premature death. Treatment options include surgical and percutaneous transcatheter closure. Surgical closure is safe and effective and when done before age 25 years is associated with normal life expectancy. Transcatheter closure offers a less invasive alternative for patients with a secundum defect. In this review we discuss the anatomy, pathophysiology, treatment options with emphasis on technical aspects, and outcomes of atrial septal defects in adult patients. We provide in depth discussion on the clinical scenarios that are unique to this patient population, including septal defects in the constellation of pulmonary hypertension and diastolic dysfunction.
\end{abstract}

Copyright @ 2016 Science International Corp.

\section{Key Words}

Atrial septal defect • Adult congenital

\section{Conflict of Interest}

The authors have no conflict of interest relevant to this publication.

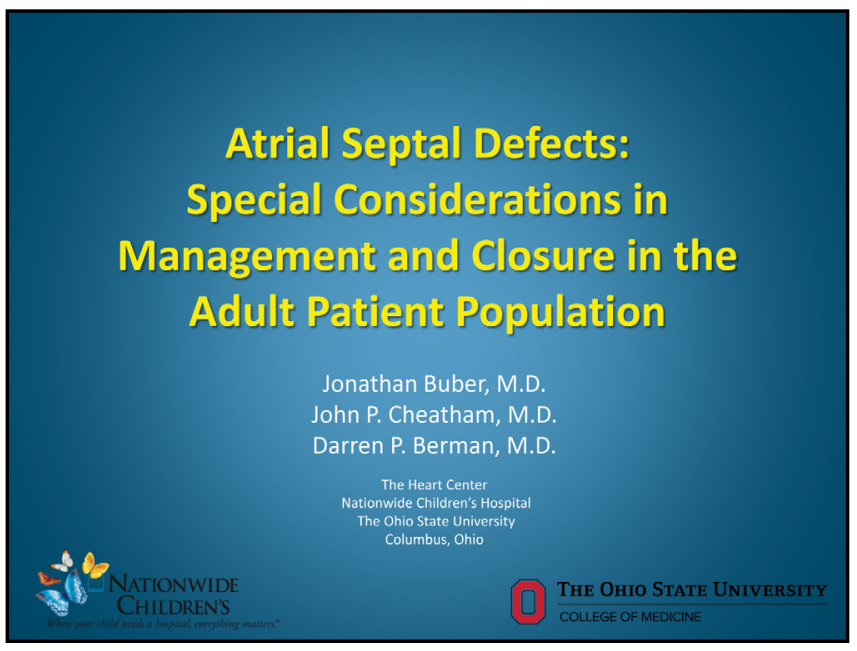

Slideshow. Click on the presentation above to view the presentation online (23.8MB). You may download the file at http://dx.doi.org/10.12945/j.jshd.2016.014.14.ppt.01.

Comment on this Article or Ask a Question

Cite this article as: Buber J, Cheatham JP, Berman DP. Atrial Septal Defects: Special Considerations in Management and Closure in the Adult Patient Population. Structural Heart Disease 2016;2(2):46. DOI: http://dx.doi.org/10.12945/j. jshd.2016.014.14 